\title{
Heart Rate Control and Perception of Exertion Towards Adolescents in Fitness Tests During Pandemic
}

\author{
Rizki Mulyawan ${ }^{1 . *}$, Yudik Prasetyo ${ }^{1}$, Fatkurahman Arjuna ${ }^{1}$, Farid Imam Nurhadi ${ }^{1}$ \\ ${ }^{1}$ Sport Science Study Program, Universitas Negeri Yogyakarta, Indonesia. \\ "Corresponding author.Email: rizkimulyawan@uny.ac.id
}

\begin{abstract}
This study aimed to control heart rate and perception of exertion during physical fitness tests, both the bleep test and the $2.4 \mathrm{~km}$ cooper test, which were carried out during the pandemic. This research was analytical observational research with a cross-sectional approach and purposive sampling technique, which obtained 43 and 37 subjects. The data collection method used capillary heart rate checking and rating perceived exertion (RPE). Furthermore, the data were analyzed using the homogeneity test, Kolmogorov-Smirnov normality test, and Pearson's Correlation test. The research results showed that the average student VO2Max on the bleep and cooper tests was $33.55 \pm 6.86$ and $33.27 \pm$ 8.38 , with no significant difference $(0.870>0.05)$ between the results of the fitness test. There was a very significant difference in heart rate as soon as possible after the test $(0.000<0.05), 15$ minutes $(0.000<0.05)$ and 30 minutes after the test $(0.000<0.05)$, while in the RPE there was only a very significant difference $(0.000<0.05)$ as soon as possible, only after the fitness test, not in other data collection times. On the other hand, there was a very significant relationship (Pearson cor., $0.703>0.05$, sig. $0.000<0.05$ ) between heart rate and perceived exertion. Therefore, the fitness of students in the low category was proven during the pandemic. There was a very significant difference in heart rate, but it did not have a significant effect on RPE. Lastly, the relationship between heart rate and RPE was very significant.
\end{abstract}

Keywords: heart rate, Borg scale, Bleep test, Cooper test, fitness.

\section{INTRODUCTION}

The pandemic changed all human habits. The new normal phase implemented in Indonesia had an impact on everyone's immune response. People who were usually actively doing sports three times a week; have turned into staying at home. As a result, there is a tendency to experience a decrease in fitness. So far, some research has explained how the pandemic had impacted the decline in physical activity patterns [1]. A study presented that tracking since before, during, and after the pandemic showed decreased physical activity patterns through tracking sports applications [2]. Then, it could risk the emergence of detraining in each individual.

Awareness of the importance of doing sports even during the pandemic clearly must remain and be consistent, even with various limitations in doing so. The use of online platforms can be one way to maintain the continuity of physical activity. The use of YouTube, for example, as long as it is still in lockdown, can be used to do exercises at home [3]. This change in habits will, of course, impact the implementation of the recommendations for physical activity from the WHO that does not work as it should. The application of exercising at least three times a week with moderate exercise intensity and the accumulation of exercise duration reached 150 minutes per week [4] seemed to be ineffective. Scientists worry that the decline in fitness levels globally is going unnoticed.

There are several aspects of health-related fitness experiencing different acute responses compared to conditions before the pandemic, including the cardiovascular system's response and aspects of perception of fatigue. Indications of fatigue will be related to the body's physiological response.

So far, many studies have only discussed how the pandemic led to changes in bodywork functions, such as reducing bodywork efficiency and recovery after 
exercise [5]. Other studies have only discussed the effect of the pandemic on physical activity levels [1], [6]. The previous research found the fitness condition, but the subject was a professional football athlete [7]. There were still a few research that discussed how the effects of new-normal life on the fitness condition of active students with an acute effect on heart rate and perception of exertion during physical activity.

It is necessary to check, especially in the age group with a small risk of disease disorders and relatively active movement ethos, namely the active adolescent group, to anticipate a mass decline in fitness. It is intended to find out earlier how the degree of fitness in the productive age group is to estimate the picture that occurs in the vulnerable age group as a continuation.

\section{METHOD}

This research was analytical observational research with a cross-sectional approach, which emphasized data observation at one time on the dependent and independent variables [8], [9].

The research subject selection technique used purposive sampling [8], with inclusion criteria (1) teenagers or active students; (2) actively exercising three times a week; (3) no injury or (4) illness in the last 3-7 days. The research subjects were 43 people during the Bleep test and 37 people during the Cooper test. The data collection method took into account health protocols due to the Covid-19 pandemic.

Heart rate data retrieval was used manually by the testers using heart rate from artery radialis that can be known through the wrist and neck area. In addition, the identification of the perception of exertion used the Borg Scale on a scale of 6-20.

Data analysis used homogeneity and normality tests, as well as independent t-test to determine the difference between the Bleep and Cooper tests.

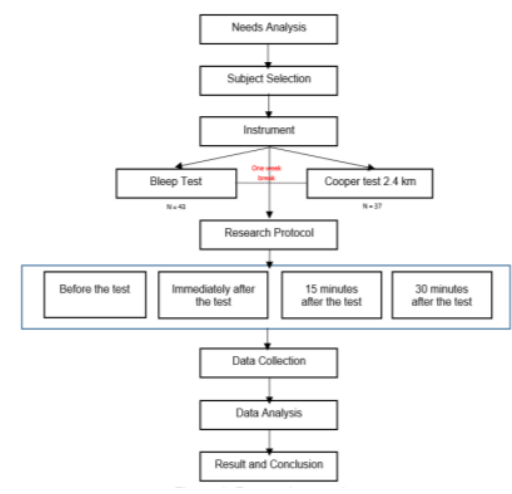

Figure 1 Research procedure.

\subsection{Research Protocol}

The interval between the Bleep test and the Cooper test was one week. The research protocol used was the same for both the Bleep and Cooper tests. The subjects were required to fast for approximately 6-8 hours since the night. The subject was recommended to drink 500 $\mathrm{ml}$ of water thirty minutes before the test [10]. After that, the fitness test (Bleep and Cooper test) was carried out, then, as soon as possible after the test, a second data collection was carried out. After the double-take was completed, the subject was instructed to take a break for a while without any fluid intake. After 15 minutes of taking a break, the third data collection was carried out. Finally, the fourth data collection was carried out 30 minutes after the test. This protocol referred to several previous studies on observational data collection and related to acute effects after exercise [10]-[12].

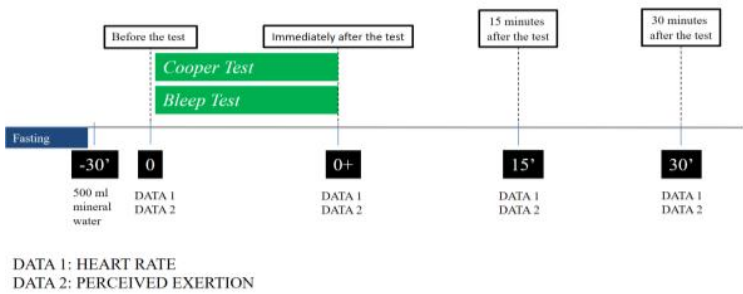

Figure 2 Research protocol

\subsection{Research Participants}

The research subjects had an average age of close to 20 years with a height and weight of $163 \mathrm{~cm}$ and 59.2 $\mathrm{kg}$, respectively.

Table 1. Average subjects description

\begin{tabular}{|l|c|}
\hline \multicolumn{1}{|c|}{ Subject Description } & Average \pm SD \\
\hline Age $($ years $)$ & $19.98 \pm 0.63$ \\
\hline Height $(\mathrm{cm})$ & $163.46 \pm 7.73$ \\
\hline Weight $(\mathrm{kg})$ & $59.22 \pm 10.97$ \\
\hline Body Fat $(\%)$ & $20.37 \pm 6.68$ \\
\hline Visceral Fat & $4.57 \pm 2.65$ \\
\hline BMI & $21.86 \pm 3.03$ \\
\hline BMR & $1384.02 \pm 222.87$ \\
\hline
\end{tabular}

\section{RESULTS}

\subsection{Results}

There was no significant difference between the bleep and cooper test $(0.870>0.05)$. The average fitness level of the students involved in this research was in a low category during the pandemic.

Table 2. Comparison of Fitness Tests

\begin{tabular}{|l|c|c|c|}
\hline $\begin{array}{c}\text { Subject } \\
\text { Description }\end{array}$ & Average \pm SD & $\begin{array}{c}\text { Average } \\
\text { Category }\end{array}$ & Sig. \\
\hline Bleep test & $33.55 \pm 6.86$ & Low & 0.870 \\
\hline Cooper test & $33.27 \pm 8.38$ & Low & \\
\hline
\end{tabular}


The heart rate was described in Table 3, whereas soon as possible after performing either the Bleep or Cooper test, the heart rate experienced a marked increase. From the statistical calculation, it was found that there was a significant difference between the Bleep and Cooper tests with a significance value of $.000<.05$ but did not apply before the test took place.

Table 3. The description of the average heart rate based on the time of taking

\begin{tabular}{|l|c|c|c|}
\hline Subject Description & $\begin{array}{c}\text { HR Bleep } \\
\text { test } \\
\text { (/minute) } \pm \\
\text { SD }\end{array}$ & $\begin{array}{c}\text { HR Cooper } \\
\text { test } \\
\text { (/minute) } \pm \\
\text { SD }\end{array}$ & Sig. \\
\hline Before the test & $80.93 \pm$ & $77.83 \pm 12.05$ & 0.303 \\
& 14.28 & & \\
\hline Immediately after the & $143.81 \pm$ & $175.40 \pm$ & $0.000^{* *}$ \\
test & 16.46 & 21.55 & \\
\hline 15 minutes after the & $115.88 \pm$ & $99.19 \pm$ & $0.000^{* *}$ \\
test & 11.88 & 22.158 & \\
\hline 30 minutes after the & $114.27 \pm$ & $95.67 \pm 18.93$ & $0.000^{* *}$ \\
test & 10.62 & & \\
\hline
\end{tabular}

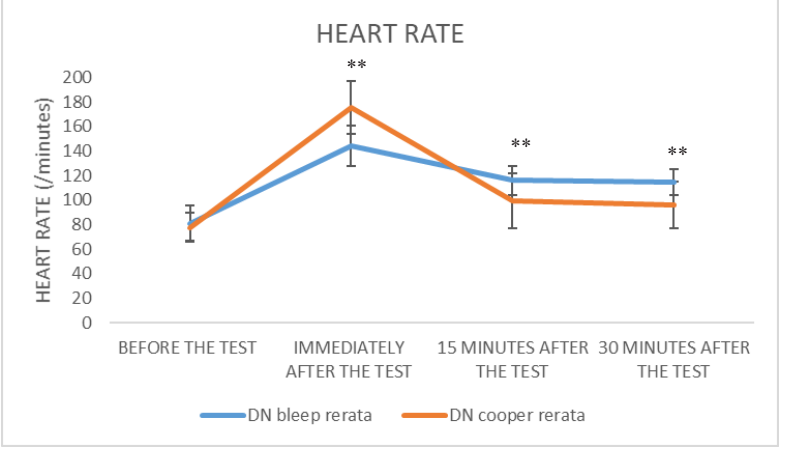

Figure 3 Comparison of heart rates based on the time of taking.

The perception of exhaustion used the Borg scale instrument showed that there was a very significant difference with a value of $.000<.05$ immediately after the test. However, there was no difference before $(.076>.05), 15$ minutes $(.191>.05)$, and 30 minutes $(.761>.05)$ after the test.

Table 4. Comparison of perception of exertion

\begin{tabular}{|l|c|c|c|}
\hline \multicolumn{1}{|c|}{ Subject Description } & $\begin{array}{c}\text { HR Bleep test } \\
\text { (/minute) } \pm \\
\text { SD }\end{array}$ & $\begin{array}{c}\text { HR Cooper } \\
\text { test (/minute) } \\
\pm \text { SD }\end{array}$ & Sig. \\
\hline Before the test & $8.35 \pm 1.70$ & $9.03 \pm 1.66$ & .076 \\
\hline Immediately after the test & $13.74 \pm 3.02$ & $16.38 \pm 1.40$ & $.000^{* *}$ \\
\hline 15 minutes after the test & $10.32 \pm 1.7$ & $10.83 \pm 1.77$ & .191 \\
\hline 30 minutes after the test & $9.05 \pm 1.79$ & $9.16 \pm 1.55$ & .761 \\
\hline
\end{tabular}

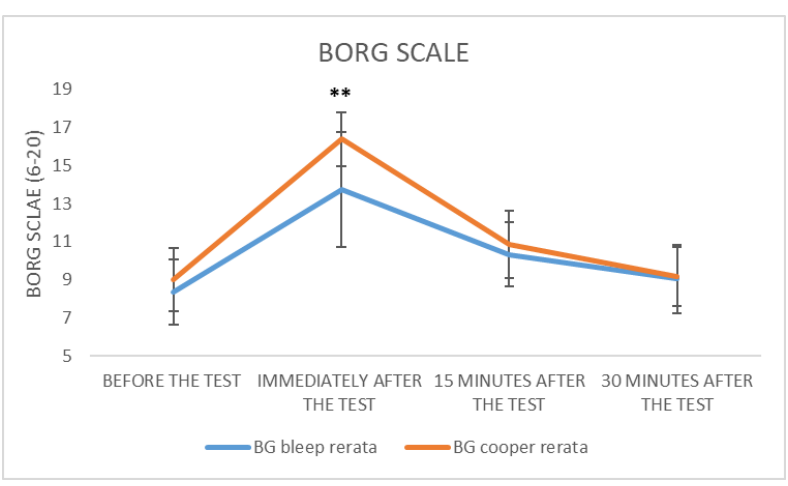

Figure 4 Differences in the Borg Scale during the Bleep and Cooper tests.

After knowing the difference between the bleep and cooper test before, as soon as possible after the test, 15 minutes and 30 minutes after the test, it turned out that there was a very significant relationship between heart rate and perceived exertion, as seen in Table 5.

Table 5. Correlation of heart rate and borg scale

\begin{tabular}{|c|c|c|}
\hline Subject Description & $\begin{array}{c}\text { Pearson } \\
\text { Correlation }\end{array}$ & Sig. \\
\hline $\begin{array}{l}\text { Correlation between Heart Rate and } \\
\text { Borg Scale }\end{array}$ & 0.703 & $0.000 * *$ \\
\hline
\end{tabular}

There was a significant relationship between heart rate and perceived exertion with a significance value of $.000<0.05$. Meanwhile, the r-table value of 0.220 was obtained four times when taking data from 80 data subjects. The results of Pearson's calculations showed $.703>.220$. The results showed that there was a relationship between variables. Therefore, it can be concluded that there was a very significant relationship between heart rate and perception of energy exertion.

\subsection{Discussion}

This research stated that the student's fitness condition was in a low category (poor). Students performed both types of physical fitness tests to measure the ability of maximal oxygen volume capacity. The intervals between one test and another were separated by a period of one week. There were some significant differences in heart rates, especially when performing the Bleep and Cooper tests. There was a significant difference when collecting data as soon as possible, 15 minutes and 30 minutes after the test. Field observations showed that the effort expended when conducting the Cooper test differed from the Bleep test. The subjects were forced to complete the distance without exception so that maximum effort was needed to complete a physical activity with full intensity when performing the $2.4 \mathrm{~km}$ Cooper test. Meanwhile, it was seen from observations during the Bleep test that the maximal effort was indeed made from each level and shuttle. However, when the subject felt unable to follow on the subject's consciousness, they could choose to 
force continue or stop doing it. Differences in implementation protocols were one of the causes of differences, even though both aimed to know the degree of fitness of each individual.

Heart rate is one of the predictors of exercise intensity. If described through exercise zones, usually by adopting the use of maximum heart rate, a heart rate below 130 per minute falls into the low-intensity category, a heart rate between $130-160$ plunges into a moderate intensity, then above 160 beats per minute is categorized into in high intensity [13]. More specifically, other recommendations in determining the intensity are recommended to apply moderate intensity in the range of $64 \%-75 \%$ of the maximum heart rate and high intensity in the field of $76 \%-95 \%$ of the entire heart rate [14], [15].

Adapting heart rate to high-intensity exercise on trained people can reduce the rate faster because of the effectiveness of the work supported by the heart muscle and a larger heart volume. Therefore, more blood can be produced in one beat without working hard [14], [16], [17].

The work of the heart rate that adapts to the ability of each individual is in line with various previous studies related to the cardiovascular system [18]. During the test, the intensity increased as the body's maximum workability must be achieved [14], It was usually indicated by lactate as an indicator of fatigue [19]. Maximum aerobic ability and heart rate variation increased, followed by physical activity interventions [20].

Perceived energy drain or commonly called Rating Perceived Exertion (RPE), is a step to measure the level of intensity of physical activity. It is based on the physical sensations experienced by a person during physical activity, including increased heart rate, increased respiratory rate, increased sweating, and muscle fatigue. Although this measurement is subjective, it ranges from a scale of 6 to 20, which is expected to provide a reasonable estimate of the heart rate during physical activity [21]. The RPE instrument using the Borg scale is considered very affordable, practical, and valid to control and determine exercise intensity, regardless of gender, age, equipment used during exercise, and level of physical activity [22].

Based on the correlation between heart rate and the Borg scale, the work effectiveness of the 6-20 ranking on the Borg scale helped control exercise intensity [23]. Examples of application in the field would relate to, for example, the longer warm-up turned out to make players feel more tired than the shorter warm-up duration [24]. The previous studies discussed the relationship between the heart rate and RPE that was not very significant in qualifying the training load [25], However, it was effective in controlling exercise intensity [23].

\section{CONCLUSION}

The components of physical fitness in sports students were in a low category, both Bleep and Cooper tests. Meanwhile, there was a significant relationship between heart rate and perceived exertion.

\section{ACKNOWLEDGMENTS}

Research Group Grant Number T/118/UN34.16/HK.03/2021 from Universitas Negeri Yogyakarta supported this work.

\section{REFERENCES}

[1] M. Brown, D. O’Connor, C. Murphy, M. McClean, A. McMeekin, and G. Prue, "Impact of COVID-19 on an established physical activity and behaviour change support programme for cancer survivors: An exploratory survey of the Macmillan Move More service for Northern Ireland," Support. Care Cancer, 2021, doi: 10.1007/s00520-021-06165-1.

[2] H. McCarthy, H. W. W. Potts, and A. Fisher, "Physical activity behavior before, during, and after COVID-19 restrictions: Longitudinal smartphone-tracking study of adults in the United Kingdom,” J. Med. Internet Res., 2021, doi: $10.2196 / 23701$.

[3] M. Adamakis, "Physical activity, sleep and weight management in the covid-19 era: A case report," J. Phys. Educ. Sport, 2021, doi: 10.7752/jpes.2021.01008.

[4] World Health Organization, WHO Guidelines on physical activity, sedentary behaviour. 2020.

[5] O. Mozolev et al., "Motor activity and physical abilities of students in the conditions of restrictions of COVID-19," Int. J. Hum. Mov. Sport. Sci., 2021, doi: 10.13189/saj.2021.090306.

[6] N. Zenic et al., "Levels and changes of physical activity in adolescents during the COVID-19 Pandemic: Contextualizing urban vs. Rural living environment," Appl. Sci., 2020, doi: 10.3390/APP10113997.

[7] E. Rampinini, F. Donghi, M. Martin, A. Bosio, M. Riggio, and N. A. Maffiuletti, "Impact of COVID-19 Lockdown on Serie A Soccer Players' Physical Qualities," Int. J. Sports Med., 2021, doi: 10.1055/a-1345-9262. 
[8] J. W. Creswell and J. D. Creswell, "Research Design: Qualitative, Quantitative, and Mixed Methods Approaches - John W. Creswell, J. David Creswell - Google Books," SAGE Publications, Inc. 2018.

[9] D. Ary, L. C. Jacobs, C. Sorensen, and A. Razavieh, Introduction to Research in Education, Wadsworth Cengage Learning. 2010.

[10] R. Syafriani, E. Y. Sukandar, T. Apriantono, and J. I. Sigit, "The Effect of Coconut Water (Cocos Nucifera L.) and an Isotonic Drink on the Change of Heart Rate Frequency in the Rats Induced Hypertension," Procedia Chem., vol. 13, pp. 177-180, 2014, doi: 10.1016/j.proche.2014.12.023.

[11] K. Dow, R. Pritchett, K. Roemer, and K. Pritchett, "Chocolate milk as a post-exercise recovery aid in division II collegiate volleyball players," Women Sport Phys. Act. J., vol. 27, no. 1, 2019, doi: 10.1123/wspaj.2018-0012.

[12] K. Pritchett and R. Pritchett, "Chocolate milk: A post-exercise recovery beverage for endurance sports," Med. Sport Sci., 2013, doi: $10.1159 / 000341954$.

[13] D. L. S. Sharon A. Plowman, Exercise Physiology for Health, Fitness, and Performance. 2017.

[14] J. P. Porcari, C. X. Bryant, and F. Comana, Exercise Physiology (Foundations of Exercise Science) 1st Edition. 2015.

[15] B. A. Roy, "Monitoring your exercise intensity," ACSM's Health and Fitness Journal. 2015, doi: 10.1249/FIT.0000000000000128.

[16] W. L. Kenney, J. H. Wilmore, and D. L. Costil, Physiology of Sport and Exercise. Sixth Edition. 2015.

[17] J. E. Hall and M. E. Hall, "Guyton and Hall: Textbook of Medical Physiology 14th Edition," Elsevier, 2021.

[18] G. A. Brown, M. W. Ray, B. M. Abbey, B. S. Shaw, and I. Shaw, "Oxygen consumption, heart rate, and blood lactate responses to an acute bout of plyometric depth jumps in college-aged men and women," J. Strength Cond. Res., 2010, doi: 10.1519/JSC.0b013e3181b22b63.

[19] B. Akinci, Y. Zenginler Yazgan, and T. Altinoluk, "The effectiveness of three different recovery methods on blood lactate, acute muscle performance, and delayed-onset muscle soreness: A randomized comparative study," $J$. Sports Med. Phys. Fitness, 2020, doi: 10.23736/S0022-4707.19.10142-9.

[20] V. K. Sharma, S. K. Subramanian, K. Radhakrishnan, R. Rajendran, B. S. Ravindran, and V. Arunachalam, "Comparison of structured and unstructured physical activity training on predicted VO2max and heart rate variability in adolescents - A randomized control trial," $J$. Basic Clin. Physiol. Pharmacol., 2017, doi: 10.1515/jbcpp-2016-0117.

[21] G. Borg, Borg's perceived exertion and pain scales. 1998.

[22] J. Scherr, B. Wolfarth, J. W. Christle, A. Pressler, S. Wagenpfeil, and M. Halle, "Associations between Borg's rating of perceived exertion and physiological measures of exercise intensity," Eur. J. Appl. Physiol., 2013, doi: 10.1007/s00421-012-2421-x.

[23] R. Canário-Lemos et al., "Are heart rate and rating of perceived exertion effective to control indoor cycling intensity?," Int. J. Environ. Res. Public Health, 2020, doi: 10.3390/ijerph17134824.

[24] J. Yanci, J. Iturri, D. Castillo, M. Pardeiro, and F. Y. Nakamura, "Influence of warm-up duration on perceived exertion and subsequent physical performance of soccer players," Biol. Sport, 2019, doi: 10.5114/biolsport.2019.81114.

[25] J. A. Rodríguez-Marroyo and C. Antoñan, "Validity of the session rating of perceived exertion for monitoring exercise demands in youth soccer players," Int. J. Sports Physiol. Perform., 2015, doi: 10.1123/ijspp.2014-0058. 